\title{
Unusual Distal LAD Spiral Dissection in Young Male
}

\author{
Ibrahim Osman, MD, OlisaemekaAchike, MD, Dan Le,MD, Assad Movahed, MD \\ East Carolina University- Brody School of Medicine \\ Department of Cardiovascular Sciences, East Carolina Heart Institute, Greenville, NC, USA \\ osmani@ecu.edu
}

\begin{abstract}
Spontaneous coronary artery dissection (SCAD) is a rare cause of acute coronary syndrome (ACS). The true incidence of SCAD is underestimated. The pathophysiology is still not fully understood and its management can be challenging.Patients may present with a broad spectrum of clinical scenarios, ranging from angina pectoris to myocardial infarction, cardiogenic shock, and sudden death. Standard therapy has not been established; current treatments range from conservative management to percutaneous revascularization or coronary artery bypass surgery. SCAD greatly mimics ACS, and this diagnosis should be considered when evaluating young patients who present with ACS with or without classical risk factors for coronary artery disease.

We report a case of a 39-year old man who presented with diabetic ketoacidosis (DKA) and presentation of ACS. He had multiple risk factors for CAD such as a smoking history of two pack-years, hypertension and poorly controlled diabetes mellitus type I. Once the clinical findings were suggestive of acute anterior and inferior myocardial infarction, the patient underwent emergent cardiac catheterization, which revealed distal left anterior descending coronary artery dissection.

Conclusion: This case highlights the fact that although SCAD is a rare entity, it is increasingly being recognized as a significant cause of ACS. Urgent angiography should be considered if SCAD is suspected, because early diagnosis and appropriate management significantly improves the outcome.
\end{abstract}

\section{INTRODUCTION}

Spontaneous coronary artery dissection (SCAD) is an extremely rare cause of acute coronary syndrome (ACS), with a reported angiographic incidence of $0.1-1.1 \%(1,2)$. Patients may present with a broad spectrum of clinical scenarios, ranging from angina pectoris to myocardial infarction, cardiogenic shock, and sudden death (3). It occurs predominantly in women, with a female: male ratio of 2:1. The majority of patients are young adults, with amean age of 46 years for men and 38 years forwomen (1). In $>70 \%$ of cases, the diagnosis is made on postmortem examination $(4,5)$. SCAD can present with sudden death and, unless an autopsy is performed, the true number of cases will remain underestimated. Other factors leading to underestimation of its prevalence are false-negative results on coronary angiography and spontaneous closure of the dissection (6).

We report a case of a 39-year-old male who presented with chest pain for 4 days and electrocardiographic findings of anterior myocardial infarction. He was found on angiography to have SCAD of the distal left anterior descending (LAD) coronary artery.

\section{CASE}

A 39-year oldCaucasian male with past medical history of hypertension, diabetes mellitus type Iandtobacco usepresented with multiple complaints includingnon-exertional substernal chest pain,epigastric abdominal 
Unusual Distal Lad Spiral Dissection in Young Male

pain and emesis ongoing for 3 days.Chest painhad been preceded by dyspnea on exertion over a period of one week. Physical examination showed mild epigastric tenderness. Blood pressure was 119/79 mm Hg, with a pulse rate of 111 beats per minute and respiratory rate of 13 breaths per minute. His temperature was $36.7^{\circ} \mathrm{C}$ $\left(98^{\circ} \mathrm{F}\right)$. Oxygen saturation was $100 \%$ on room air. Electrocardiography (ECG) showed sinus tachycardia, left posterior fascicular block, ST elevation in the anterior and inferior leads(Figure 1). Transthoracic Lab work revealedelevated cardiac troponin $2.95 \mathrm{ng} / \mathrm{mL}$, blood glucose of $984 \mathrm{mg} / \mathrm{dL}, \mathrm{HbA} 1 \mathrm{c}=10.7$ \%, creatinine 1.73 $\mathrm{mg} / \mathrm{dl}$, hemoglobin of $8.8 \mathrm{~g} / \mathrm{dL}$, Sodium level $124 \mathrm{mEq} / \mathrm{L}$ and Potassium level of $6.7 \mathrm{mEq} / \mathrm{L}$. Arterial blood gas showed pH of 7.20, bicarbonate level $9 \mathrm{mEq} / \mathrm{L}, \mathrm{PCO} 214 \mathrm{~mm} \mathrm{Hg}$. He was admitted to intensive care unit for diabetic ketoacidosis and a stat cardiology consult was placed. Stat bedside echocardiography showed absence of thickening of the apex and periapical region, gigantoid, multilobar, complex, separate pendular mobile thrombi (Figure 2a to 2e). Urgent coronary angiography showed total occlusion of distal portion of LAD artery with spiral dissection of LAD (figure 3) thought to be subacute or chronic, given collateral branches from diagonal branch supplying cardiac apex.

\section{Figure Legend 1}

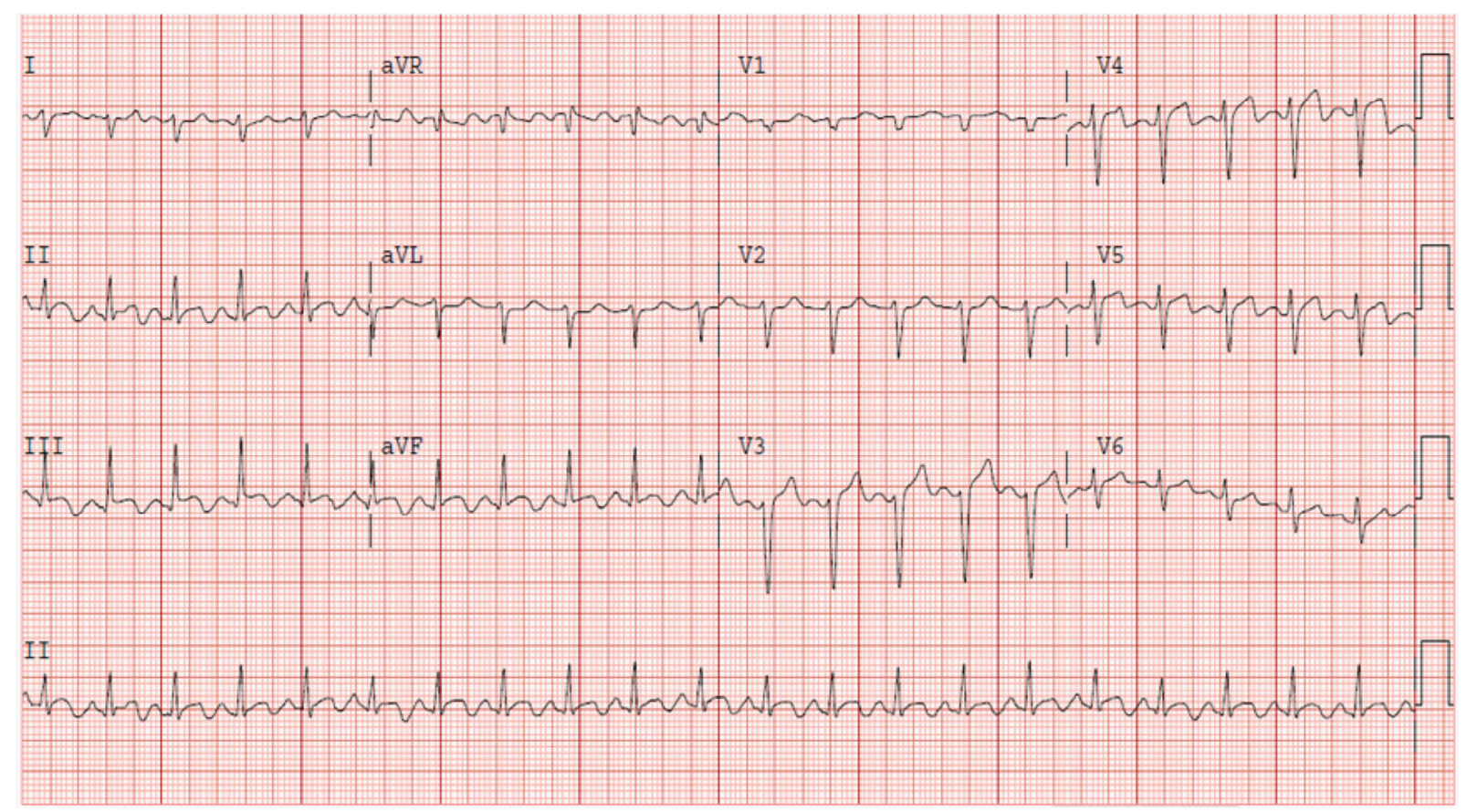

Electrocardiogram (ECG) showing a sinus tachycardia, acute anterior and inferior infarct, left posterior fascicular block (LPFB) and prolonged QT interval

He received loading dose of aspirin $325 \mathrm{mg}$ then $81 \mathrm{mg}$ daily, loading dose of clopidogrel $600 \mathrm{mg}$ once and then $75 \mathrm{mg}$ daily, unfractionated heparin intravenous bolus of 60 units per kilogram then 12 units per kilogram intravenous infusion, atorvastatin $80 \mathrm{mg}$ nightly and metoprolol tartrate $25 \mathrm{mg}$ twice daily. He was placed on Warfarin with INR target of 2-3 given his left ventricular thrombus. He was also placed on intravenous insulin per diabetic ketoacidosis protocol. Afterwards hospital course was stable. He was discharged home with plan for outpatient cardiology follow up. 
Unusual Distal LAD Spiral Dissection in Young Male

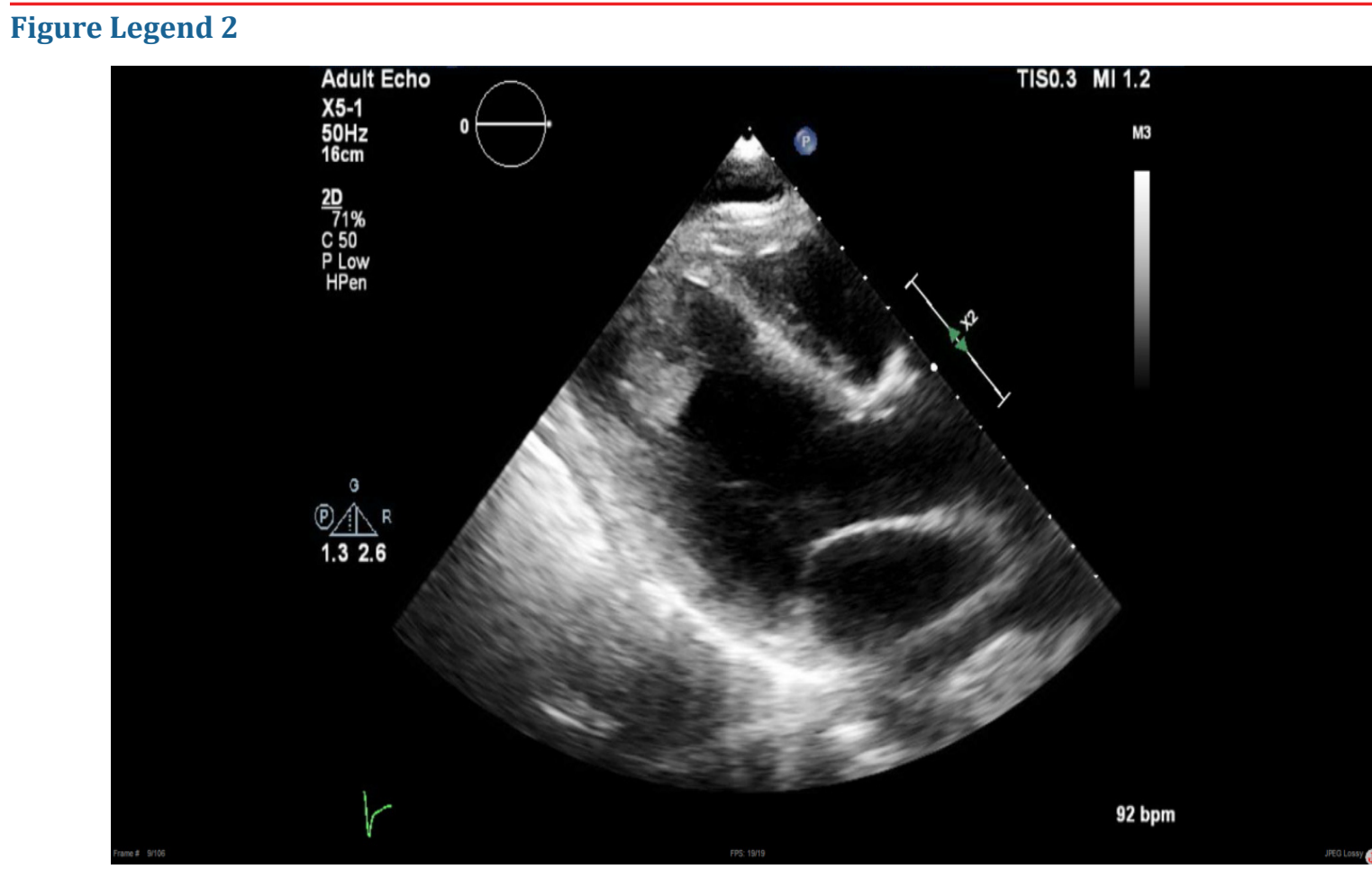

Parasternal long axis

Figure Legend 3

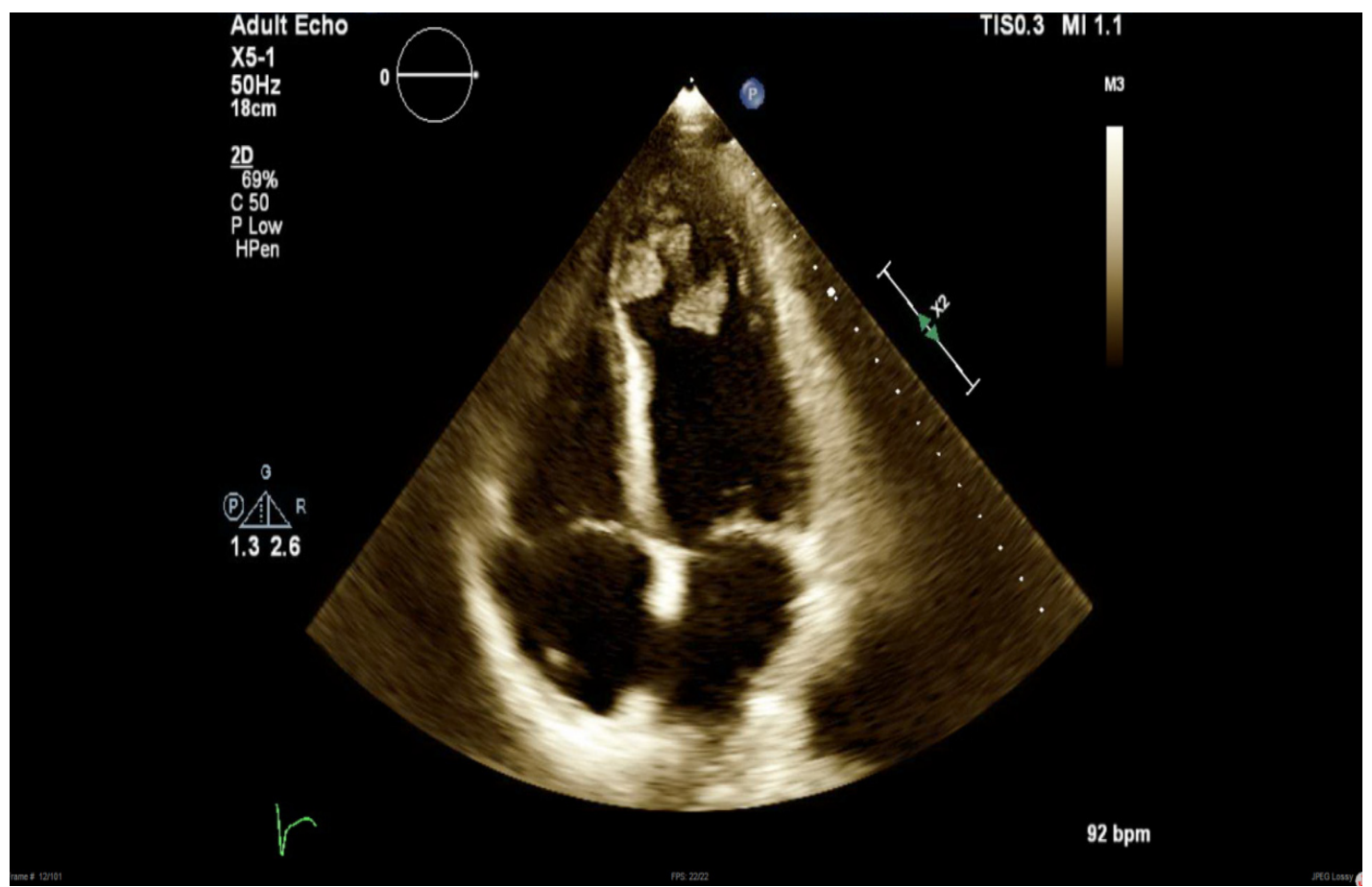

Apical 4 chambers

American Research Journal of Clinical Case Reports 
Unusual Distal LAD Spiral Dissection in Young Male

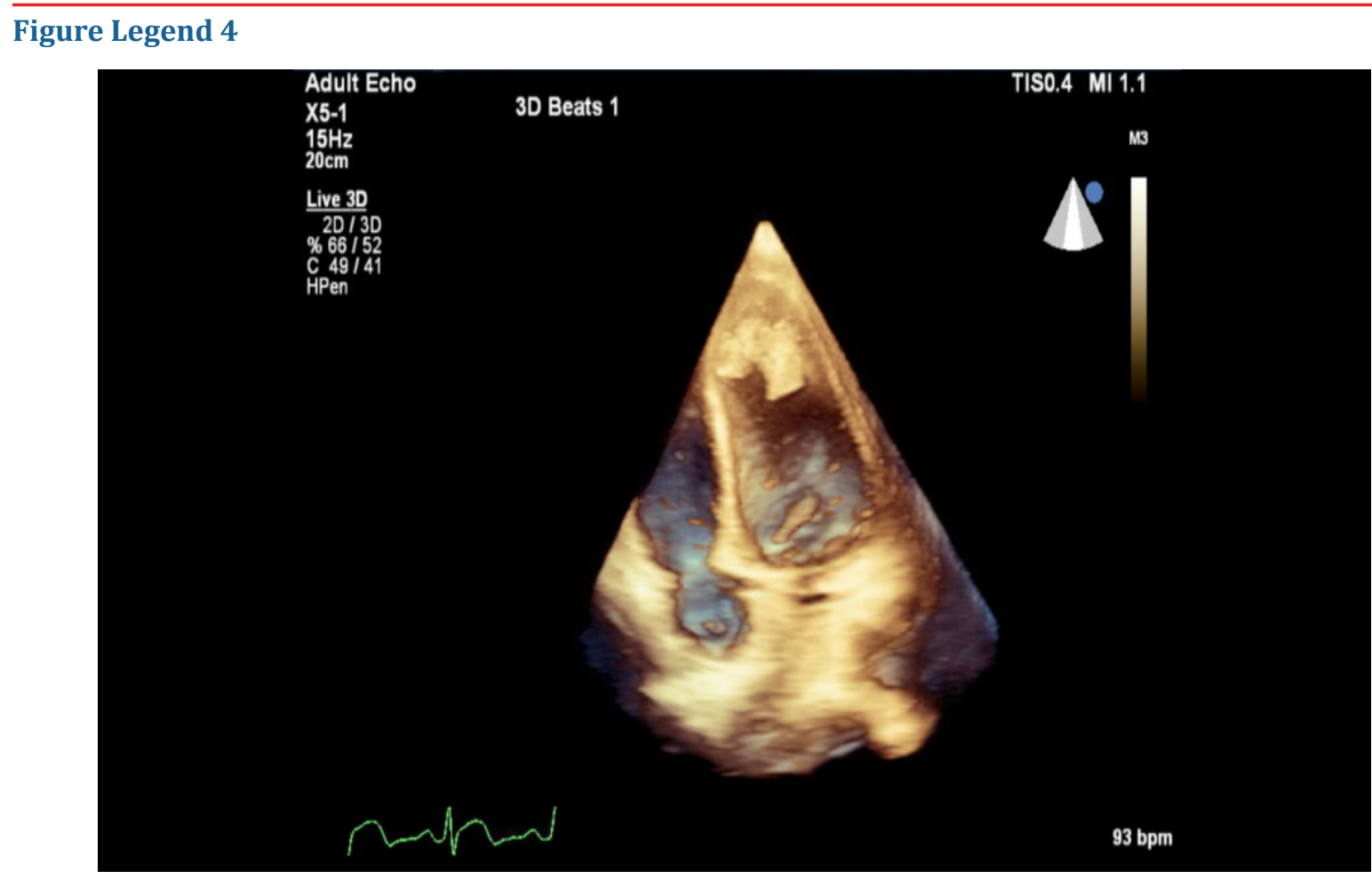

3 dimensional apical 4 chambers

Figure Legend 5

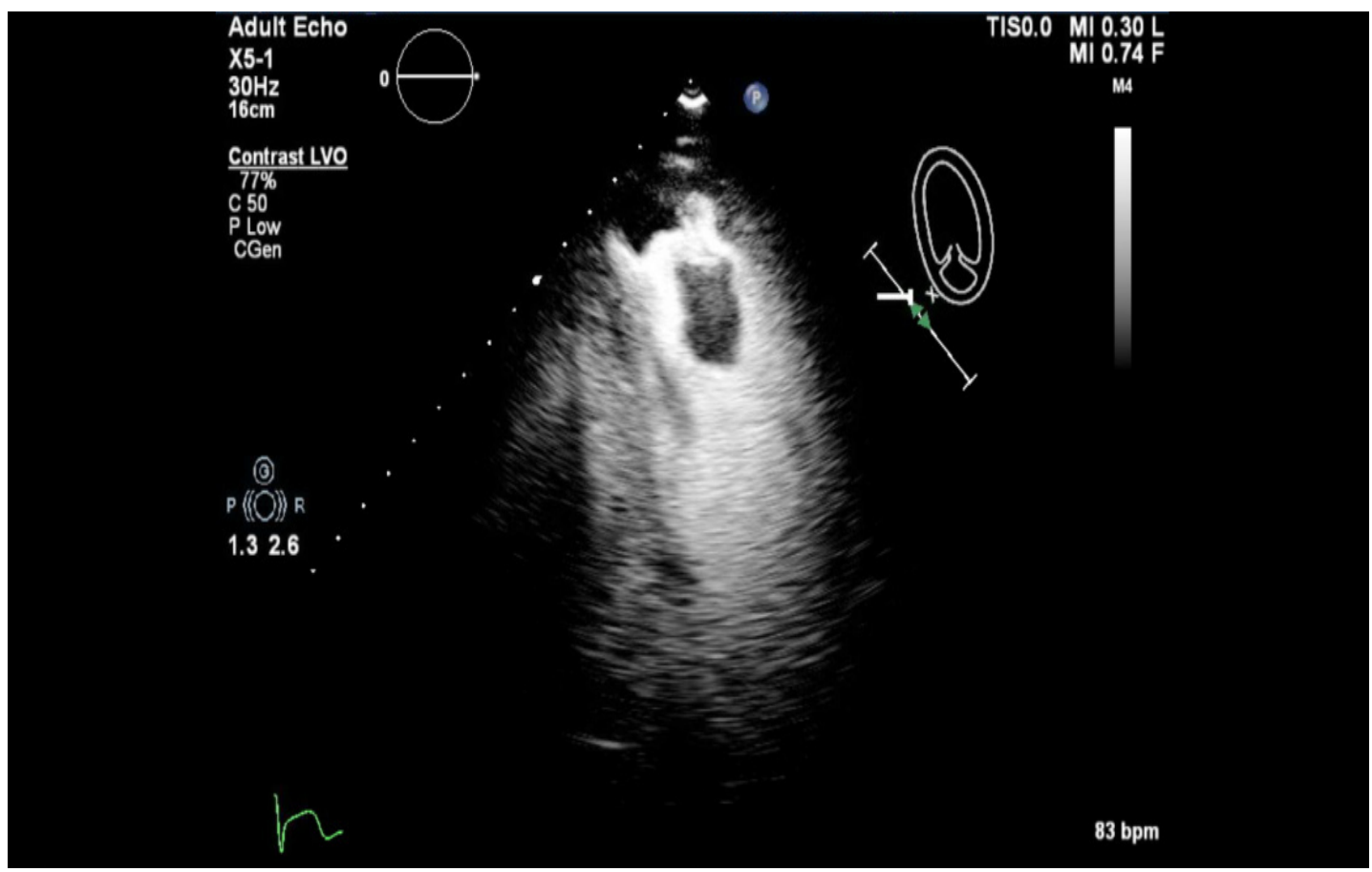

Definity study

American Research Journal of Clinical Case Reports 


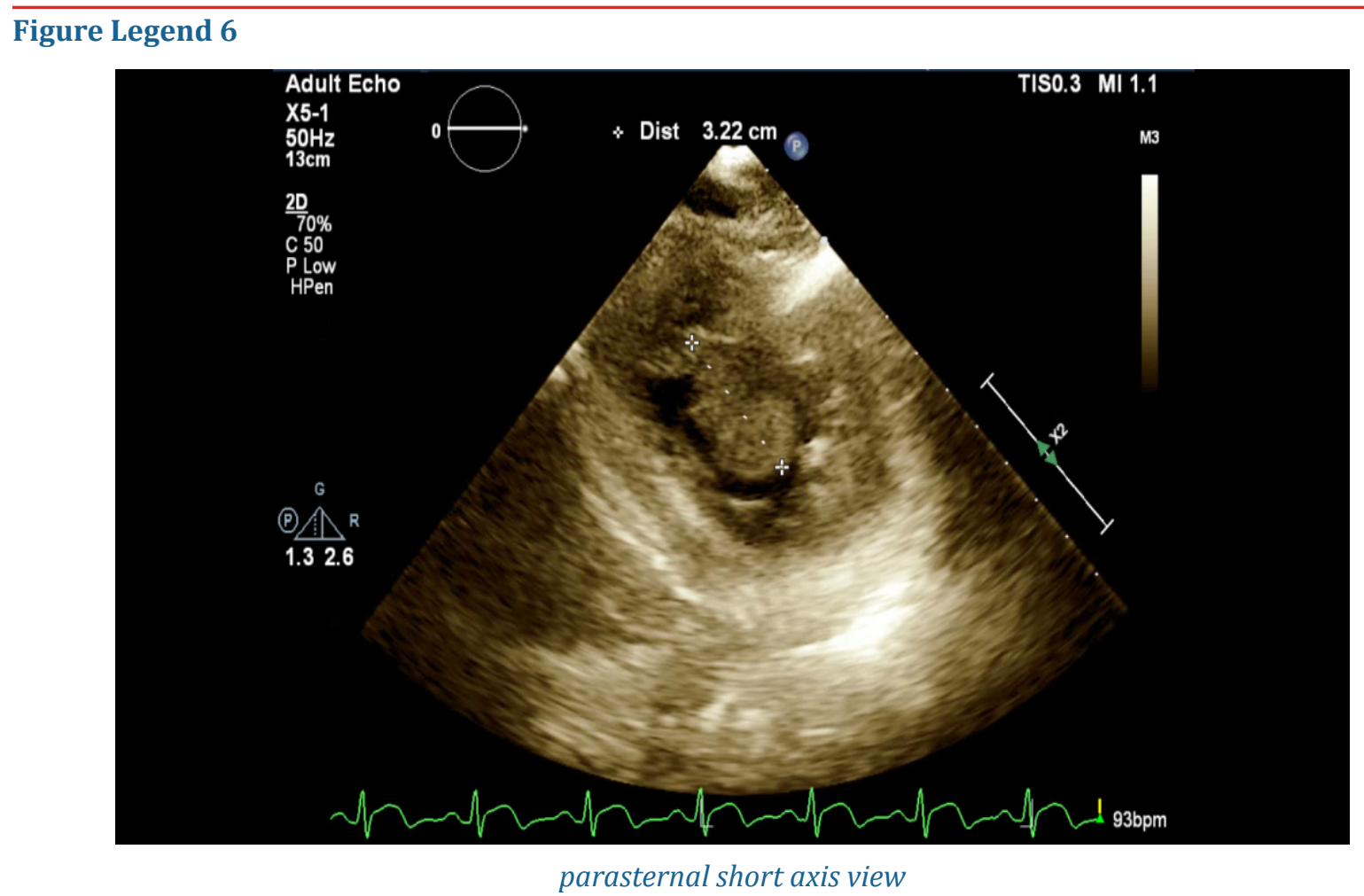

Figure (2a to 2e): Transthoracic echocardiogram multiple views showing LV thrombus

\section{DISCUSSION}

Primary SCAD is a rare cause of acute myocardial infarction and is associated with a high mortality rate of about $50 \%$. It has been observed in four groups of patients: those with coronary atherosclerosis, women in peripartum and early postpartum period, women taking oral contraceptives, and lastly in an inhomogeneous group labelled "idiopathic" as illustrated by this case due to there being no obvious associated factors. (1).

SCAD is defined as a spontaneous separation of the coronary artery wall that is not iatrogenic or related to trauma. As such, dissections due to blunt trauma, surgical instruments, or those that are catheter-induced are not deemed to be SCAD. Furthermore, contemporary usage of the term SCAD is typically reserved for the nonatherosclerotic variant, and most modern series exclude SCAD due to atherosclerotic coronary artery disease.

SCAD was previously, incorrectly believed to be very rare and to be frequently associated with pregnancy. Unfortunately, the true incidence and prevalence of SCAD in the general population is unknown due to significant under-diagnosis of this condition. SCAD affects women in $>90 \%$ of cases with rate of 9 to 1 ratio (female:male). In contemporary series that excluded patients with atherosclerotic causes, women accounted for $92 \%$ to $95 \%$ of the population with $\operatorname{SCAD}(12,15,16,18-20)$. The reported mean age ranged from 44 to 55 years in contemporary series, reflecting a relatively young to middle-age population $(12,15,16,18-21)$.

The arterial dissection with SCAD can occur within or between any of the 3 layers (intima, media, or adventitia) of the coronary artery wall. Two potential mechanisms for the initiation of arterial wall separation have been proposed (23). The first is the intimal tear hypothesis, in which a primary disruption in the intimal-luminal interface creates an entry point for intramural hematoma (IMH) accumulation inside the false lumen, leading to separation of the arterial wall. The second is the medial hemorrhage hypothesis, in which a hemorrhage into the 


\section{Unusual Distal LAD Spiral Dissection in Young Male}

arterial wall is the primary mechanism, perhaps due to spontaneous rupture from the increased density of the vasa vasorum. This creates a false lumen in the vessel, which is further expanded by bleeding, accumulation, and enlargement of the hematoma. This causes further separation of the dissected layers, leading to compression of the true lumen.Subsequently, this results in myocardial ischemia or infarction (7).

\section{Figure Legend 7}

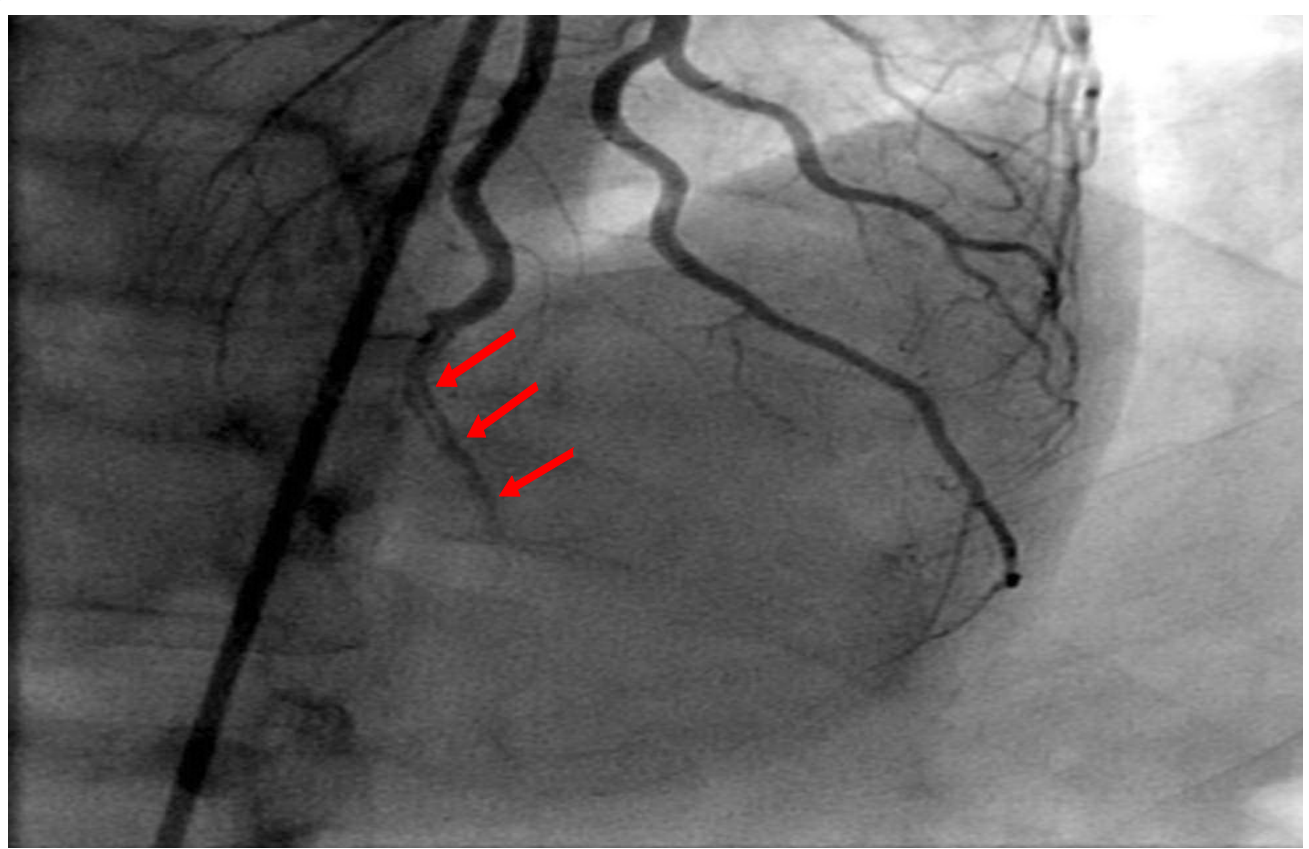

Transthoracic echocardiogram 4 chambers apical view showing LV thrombus

\section{Figure Legend 8}

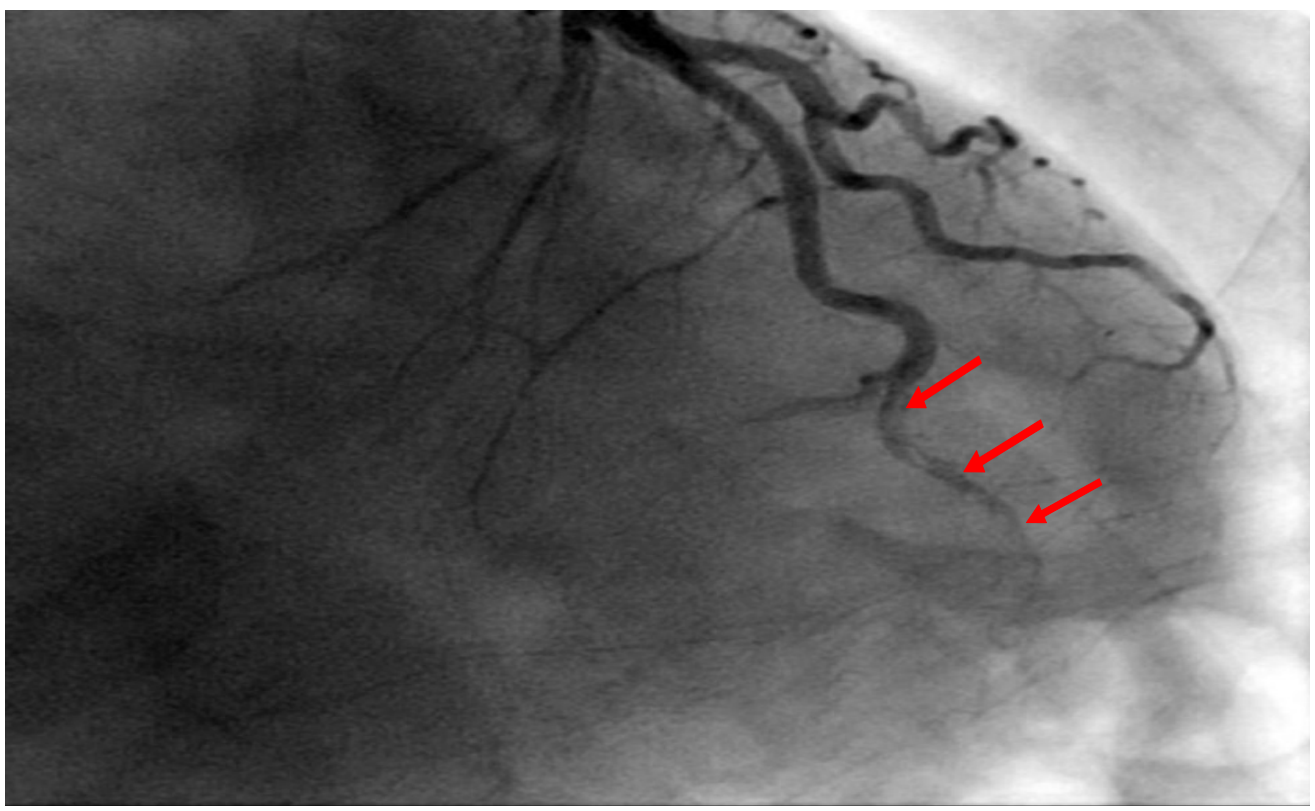

Transthoracic echocardiogram parasternal short axis view showing LV thrombus 
Unusual Distal LAD Spiral Dissection in Young Male

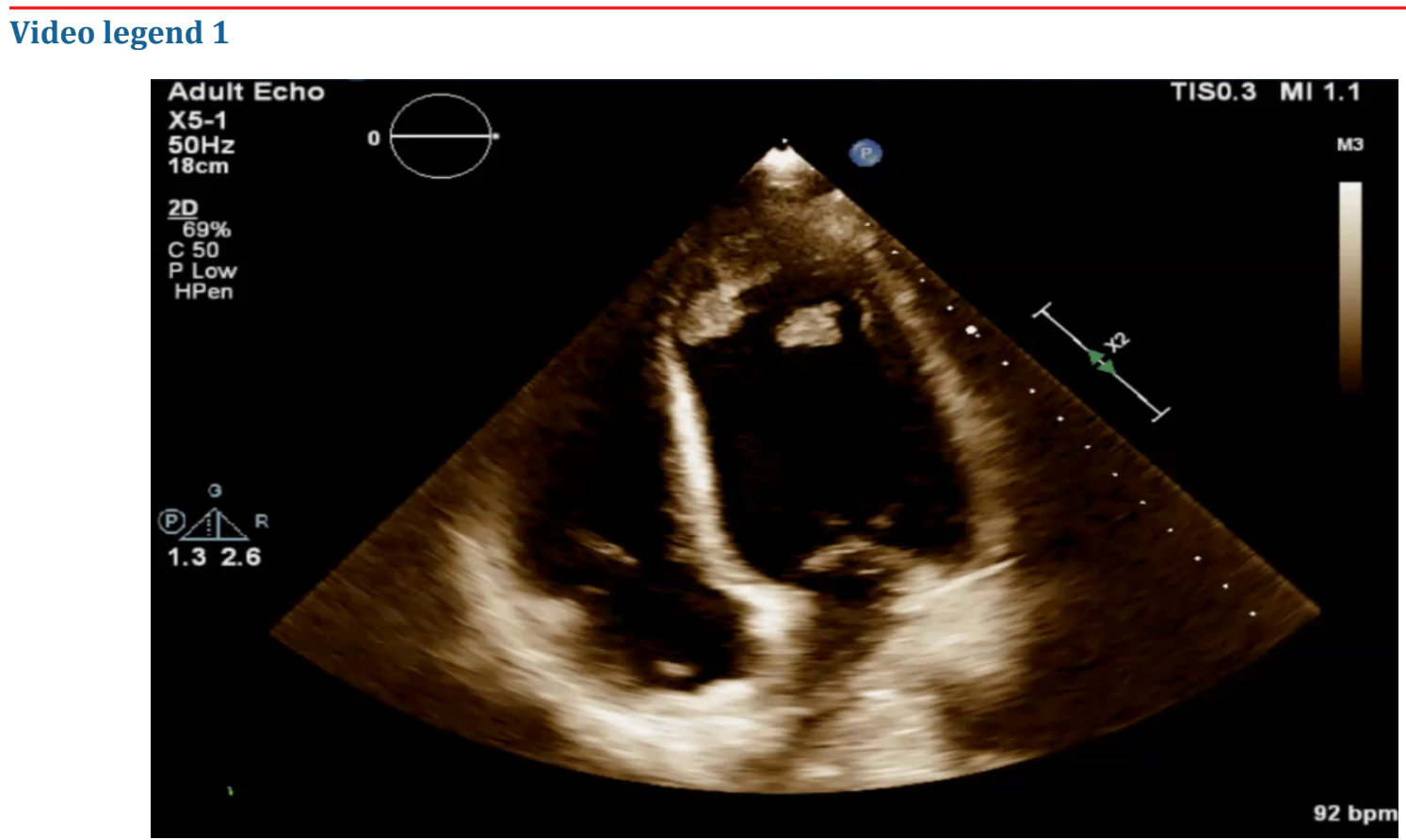

Video legend 2

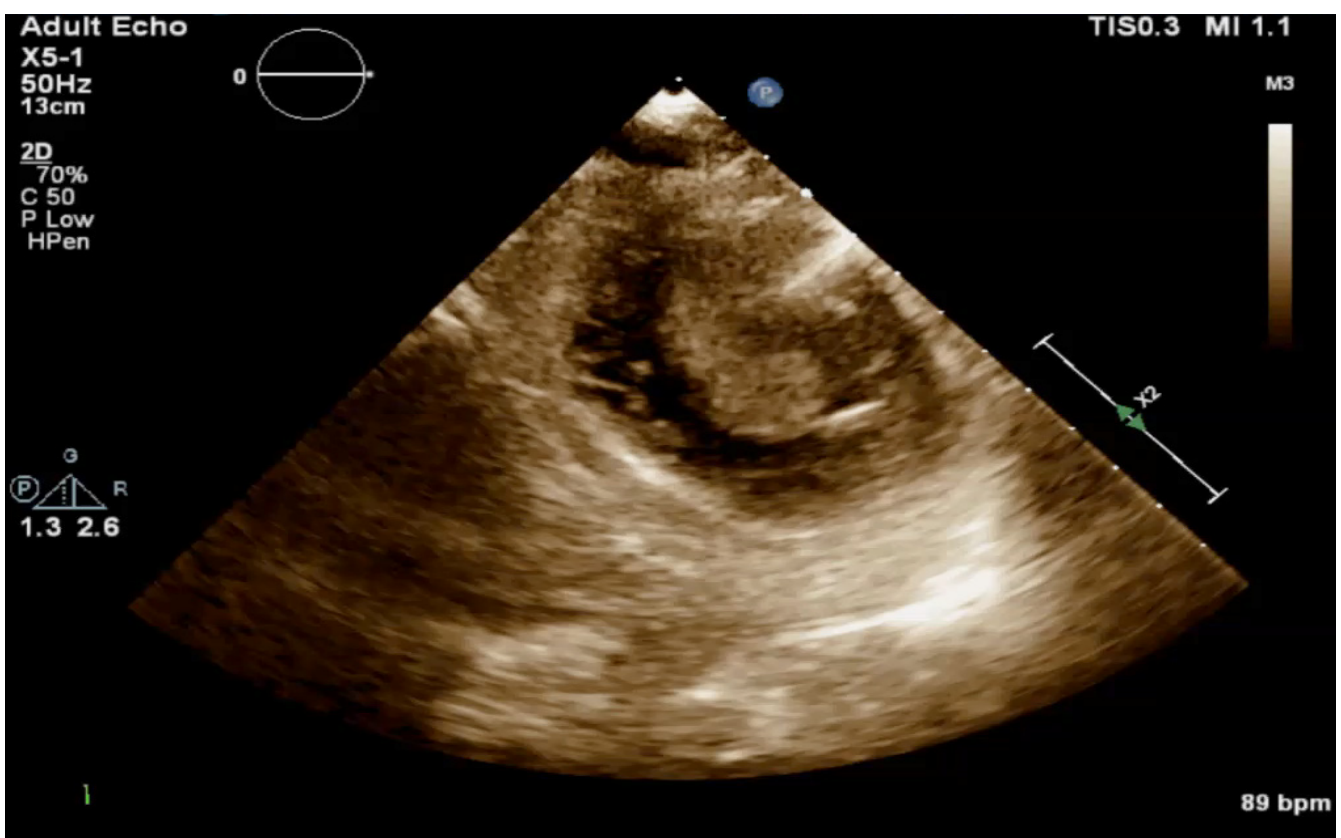

Coronary angiogram showing intimal dissection flap with preserved flow in distal LAD (Arrows)

The underlying etiology of SCAD appears to be multifactorial. There is often an associated underlying predisposing arteriopathy, which may be compounded by a precipitating stressor (Intense exercise, emotional stress, hormonal therapy, Valsava-type activities and recreational drugs) culminating in the phenotypic expression of SCAD. Many potential predisposing nonatheroscleroticarteriopathies for SCAD have been reported. The most dominant association reported is fibromuscular dysplasia (FMD), in addition to, pregnancy, connective tissue 


\section{Unusual Distal LAD Spiral Dissection in Young Male}

disease, systemic inflammatory disease, coronary vasospasm or idiopathic, cyclosporine therapy, and heavy exercise (12). A hereditary factor has been discussed previously (17).

There is a wide spectrum of clinical presentations and severity of SCAD. Almost all patients with SCAD present with ACS and elevation of cardiac enzymes $(15,17,18,21)$. The proportion who presented with STEMI varied widely in different series, ranging from $24 \%$ to $87 \%$ (15-21). A small proportion can be complicated with ventricular arrhythmias (3\% to $10 \%)(15-17)$, cardiogenic shock $(<3 \%)(15,17)$, or sudden cardiac death $(<1 \%)$, although this presentation may be underestimated (22).

The right coronary artery (RCA) is most commonly involved in men (67\%), whereas the LAD artery and the left circumflex artery are more commonly involved in women (87\%). However, irrespective of gender, overall, the LAD is affected in $75-80 \%$ of the cases, and the RCA is affected in only $20 \%$ of cases (4). The usual location of the dissection is in the proximal portion of a single coronary artery, but involvement of multiple arteries has been described $(8,9)$. Concerning the prognostic correlations, left main or LAD disease, and multi-vessel dissection tend to indicate a poor outcome, commonly resulting in sudden death or extensive myocardial infarction (6). Our case is uncommon presentation as SCAD occurred in the distal portion of the LAD, and caused ST-segment elevation seen on the inferolateral leads on ECG.

Per previous reported literature, in rare instances, acute inferior myocardial infarction may result from occlusion of the recurrent LAD branch, which is the terminal portion of a "wraparound" LAD. Consequently, besides a wraparound LAD, a more distally occluded LAD is also thought to be a prerequisite for an isoelectric or even elevated ST segment inferiorly (10).

Accurate and early diagnosis of SCAD is important because the management and investigation of SCAD is different from atherosclerotic disease. Coronary angiography is widely available and is the first-line imaging for patients presenting with ACS. However, coronary angiography has significant limitations in diagnosing SCAD because it is a 2-dimensional luminogram that does not image the arterial wall. Dedicated intracoronary imaging (optical coherence tomography [OCT] and intravascularultrasound [IVUS]) that images the arterial wall layers improves SCAD diagnosis, but it is not as widely available and is associated with additional risks and costs. Thus, coronary angiography remains instrumental in SCAD diagnosis, and angiographers should gain familiarity with the angiographic variants of SCAD. Both OCT and IVUS provide complementary details to diagnose SCAD, which requires the presence of IMH or a double lumen. OCT has a superior spatial resolution of 10 to $20 \mathrm{~mm}$ versus IVUS, with lower resolution $(\sim 150 \mathrm{~mm})$, but better penetration.

The optimal management of SCAD remains undetermined because no randomized trials have compared medical therapies or revascularization strategies, unlike atherosclerotic disease. Standard guideline indicated medical therapies administered for ACS have not been specifically studied for SCAD, and it is unclear if they are beneficial in this unique population (25). Current recommendations on management are largely used on the basis of expert opinions from observational series $(11,24,26)$. Medical therapy includes Beta-Blockade, dual antiplatelet therapy (DAPT) could be empirically beneficial, the role of novel P2Y12 antagonists (Ticagrelor and Prasugrel) for SCAD management is undefined. Glycoprotein IIb/IIIa inhibitors have also not been evaluated for SCAD; however, they are not recommendedbecause of their greater potency, higher bleeding risk, and a potential risk of extending the dissection (11). The role of anticoagulation for SCAD is controversial and has not been studied. Heparin agents are routinely administered for ACS management in hospital, but the clinical benefit has not been established for SCAD. There is a potential risk of extending the dissection with anticoagulation, which is balanced by the potential benefit of resolving overlying thrombus and improving true lumen patency. Heparin 


\section{Unusual Distal LAD Spiral Dissection in Young Male}

should likely be discontinued once the SCAD diagnosis is made; however, this practice remains controversial and lacks supportive data. Thrombolytic therapy should be avoided in SCAD because there have been reports of harm and clinical deterioration due to extension of IMH and dissection $(28,29)$. The use of statins for

SCAD is controversial. A small retrospective study demonstrated potentially higher SCAD recurrence with statins (13); however, the bulk of data for ACS demonstrates significant benefit with lipid lowering, and statins are routinely recommended post-MI (27). Because of the uncertainty and the general lack of atherosclerosis in SCAD patients, statins tend to only be administered to patients with pre-existing dyslipidemia. One series reported a high rate of statin use without apparent safety concerns (18).

Following diagnosis of SCAD, it has become routine practice in several centers specializing in SCAD to screen for predisposing arteriopathies associated with SCAD.

An overall conservative approach is preferred on the basis of expert opinions derived from observational data (11,14-17). This recommendation relies on observations that SCAD arteries heal spontaneously in most cases, and that revascularization is associated with high failure rates. Nevertheless, a small proportion of patients should be considered for revascularization, including those with ongoing or recurrent ischemia, hemodynamic instability, ventricular arrhythmias, or left main dissection.

PCI should be performed in these cases if the anatomy is suitable; otherwise, CABG should be considered. Several series have reported poor technical success with PCI for SCAD related to iatrogenic dissection and propagation to distal portion (Not suitable for stenting, longer stents), difficulty advancing coronary wire into distal true lumen and risk of stent malapposition after resorption of IMH, with risk of late stent thrombosis.

Patients are advised to avoid lifting weights $>20$ pounds, and to have a low target exercise heart rate and systolic blood pressure with this program. SCAD patients who participated in the dedicated rehabilitation program had lower long-termMACEscomparedwiththosewho did not participate (31).

The in-hospital outcomes of SCAD patients are reasonably good in contemporary prospective series. Acute in-hospital mortality was $<5 \%$ in modern series, and in-hospital recurrent MI, need for urgent revascularization in conservatively managed patients, or other MACEs were $5 \%$ to $10 \%(13,15,17)$. However, following hospital discharge, a significant proportion of patients can have recurrent chest pains and MACEs. Subacute MACEs were reported in 10\% to $20 \%$ of patients at 2-year follow-up, with recurrent SCAD occurring in w15\% (15). Longer-term recurrent SCAD rates at 4 to 5 years were reported at $\sim 27 \%(16,21)$. Although overall long-term survival is good in this cohort ( $>95 \%$ ), long-term MACE rates can be high, and were reported to be $15 \%$ to $37 \%$ at 5 to 7 years and estimated at $\sim 50 \%$ at 10 years $(13,15,17,21)$. In the updated prospectively followed cohort of 280 patients, MACEs were $20.4 \%$ at a median 2.3-year follow-up; recurrent MI was $19.0 \%$, with a recurrent SCAD rate of $12.2 \%$ (30). Suchhigh long-term MACErates emphasize the importance of close follow-up of SCAD survivors by cardiovascular specialists. Of note, patients with post-partum SCAD may have worse prognoses than other SCAD cohorts. In a small retrospective series, post-partum patients had larger infarcts, lower mean left ventricular ejection fractions, and tended to have more proximal artery dissections (32).

We report an unusual case of distal LAD spiral spontaneous dissection in young male without obvious triggers, however, it is unclear whether his acute diabetic ketoacidosis and tobacco use may contribute to his presentation. With the exception of smoking, hypertension and DM, no other risk factors were present in our patient. It is noteworthy that the distal portion of the LAD is an unusual location for dissection in male like our case. PCI was not performed due to distal location of the dissection, vessel diameter $<2.5 \mathrm{~mm}$ and the risk of propagation. 


\section{Unusual Distal LAD Spiral Dissection in Young Male}

We did not use a GP IIb/IIIa inhibitor in the present patient because of clinical success with dual antiplatelet therapy and heparin. Warfarin was initiated with INR goal of 2-3. The prognosis was favorable in our case and he was discharged home in stable condition with outpatient follow up plan.

\section{CONCLUSION}

SCAD is a rare entity, often underdiagnosed, however, it is increasingly being recognized as a significant cause of ACS.It is frequently associated with predisposing and precipitating factors. Coronary angiography with IVUS or OCT remains instrumental in SCAD diagnosis.Conservative therapy is preferred, except for patients with unstable symptoms, hemodynamic instability or left main dissection. Overall survival is good; however, long-term MACEs are frequent, including recurrent SCAD, therefore, SCAD patients should be closely followed for cardiac events.

\section{REFERENCES}

1. De Maio SJ, Kinsella SH, Silverman ME. Clinical course and longterm prognosis of spontaneous coronary artery dissection. Am J Cardiol 1989;64:471-4.

2. Hering D, Piper C, Hohmann C, Schultheiss HP, Horstkotte D. Incidence, etiology and therapy of spontaneous coronary artery dissection. A prospective monocenter study of 3800 consecutive patients [German]. Z Kardiol 1998;87:961-70.

3. Dhawan R, Singh G, Fesniak H. Spontaneous coronary artery dissection: the clinical spectrum. Angiology 2002;53:89-93.

4. Nogueira de MR, de Paula MS, Vieira da Costa RL. Spontaneous coronary artery dissection - a diagnosis to be considered in young patients presenting with acute myocardial infarction. J Invasive Cardiol 2009;21:E245-7.

5. Bergen E, Huffer L, Peele M. Survival after spontaneous coronary artery dissection presenting with ventricular fibrillation arrest. EP Lab Digest. Available at: www.eplabdigest.com. Accessed January 30, 2011.

6. Oliveira SM, Goncalves A, Dias P, et al. Spontaneous coronary artery dissection: a diagnosis to consider in acute coronary syndromes. Rev Port Cardiol 2009;28:707-13.

7. Kamineni R, Sadhu A, Alpert J. Spontaneous coronary artery dissection: report of two cases and a 50-year review of the literature. Cardiol Rev 2002;10:279-84.

8. Cohen DE, Strimike CL. A case of multiple spontaneous coronary artery dissections. Catheter CardiovascInterv 2000;49:318-20.

9. Celik SK, Sagcan A, Altintig A, Yuksel M, Akin M, Kultursay H. Primary spontaneous coronary artery dissections in atherosclerotic patients. Report of nine cases with review of the pertinent literature. Eur J CardiothoracSurg 2001;20:573-6.

10. Porto I, Banning AP. Intravascular ultrasound imaging in the diagnosis and treatment of spontaneous coronary dissection with drug-eluting stents. J Invasive Cardiol 2004;16:78-80 Pub-Med

11. Saw J. Spontaneous coronary artery dissection. Can J Cardiol 2013;29:1027-33.

12. Saw J, Ricci D, Starovoytov A, et al. Spontaneous coronary artery dissection: prevalence of predisposing conditions including fibromuscular dysplasia in a tertiary center cohort. J Am CollCardiolIntv $2013 ; 6: 44-52$. 
Unusual Distal LAD Spiral Dissection in Young Male

13. Tweet MS, Hayes SN, Pitta SR, et al. Clinical features, management and prognosis of spontaneous coronary artery dissection. Circulation 2012; 126:579-88.

14. Alfonso F, Paulo M, Lennie V, et al. Spontaneous coronary artery dissection: long-term follow-up of a large series of patients prospectively managed with a "conservative" therapeutic strategy. J Am CollCardiolIntv 2012;5:1062-70.

15. Saw J, Aymong E, Sedlak T, et al. Spontaneous coronary artery dissection: association with predisposing arteriopathies and precipitating stressors and cardiovascular outcomes. CircCardiovascInterv 2014;7: 645-55.

16. Tweet MS, Eleid MF, Best PJ, et al. Spontaneous coronary artery dissection: revascularization versus conservative therapy. CircCardiovascInterv 2014;7:777-86.

17. Lettieri C, Zavalloni D, Rossini R, et al. Management and long-term prognosis of spontaneous coronary artery dissection. Am J Cardiol 2015;116: 66-73.

18. Rogowski S, Maeder MT, Weilenmann D, et al. Spontaneous coronary artery dissection: angiographic follow-up and long-term clinical outcome in a predominantly medically treated population. Catheter CardiovascInterv 2015 Dec 28 [E-pub ahead of print].

19. Rashid HN, Wong DT, Wijesekera H, et al. Incidence and characterisation of spontaneous coronary artery dissection as a cause of acute coronary syndrome - a single-centre Australian experience. Int J Cardiol 2016;202:336-8.

20. Roura G, Ariza-Solé A, Rodriguez-Caballero IF, et al. Noninvasive follow-up of patients with spontaneous coronary artery dissection with CT angiography. J Am CollCardiolImg 2015 Oct 6 [E-pub ahead of print].

21. Nakashima T, Noguchi T, Haruta S, et al. Prognostic impact of spontaneous coronary artery dissection in young female patients with acute myocardial infarction: a report from the AnginaPectoris-Myocardial Infarction Multicenter Investigators in Japan. Int J Cardiol 2016;207:341-8.

22. Hill SF, Sheppard MN. Non-atherosclerotic coronary artery disease associated with sudden cardiac death. Heart 2010;96:1119-25.

23. Maehara A, Mintz GS, Castagna MT, et al. Intravascular ultrasound assessment of spontaneous coronary artery dissection. Am J Cardiol 2002;89:466-8.

24. Yip A, Saw J. Spontaneous coronary artery dissection-a review. CardiovascDiagnTher 2015;5: 37-48.

25. Mehta LS, Beckie TM, DeVon HA, et al., American Heart Association Cardiovascular Disease in Women and Special Populations Committee of the Council on Clinical Cardiology, Council on Epidemiology and Prevention, Council on Cardiovascular and Stroke Nursing, and Council on Quality of Care and Outcomes Research. Acute Myocardial Infarction in Women: A Scientific Statement from the American Heart Association. Circulation 2016;133:916-47.

26. Alfonso F, Bastante T, Rivero F, et al. Spontaneous coronary artery dissection. Circ J 2014;78:2099-110.

27. Amsterdam EA, Wenger NK, Brindis RG, et al. 2014 AHA/ACC guideline for the management of patients with non-ST-elevation acute coronary syndromes: executive summary: a report of theAmerican College of Cardiology/American Heart Association Task Force on Practice Guidelines. J Am CollCardiol 2014;64: 2645-87.

28. Shamloo BK, Chintala RS, Nasur A, et al. Spontaneous coronary artery dissection: aggressive vs. conservative therapy. J Invasive Cardiol 2010;22:222-8. 
Unusual Distal LAD Spiral Dissection in Young Male

29. Zupan I, Noc M, Trinkaus D, et al. Double vessel extension of spontaneous left main coronary artery dissection in young women treated with thrombolytics. Catheter CardiovascInterv 2001;2001:226-30.

30. Saw J, Prakash R, Starovoytov A, et al. Cardiovascular outcomes in a large prospectively followed single-center cohort of spontaneous coronary artery dissection patients (abstr). J Am CollCardiol 2016;67:457.

31. Chou AY, Prakash R, Rajala J, et al. The first dedicated cardiac rehabilitation program for patients with spontaneous coronary artery dissection: description and initial results. Can J Cardiol 2016;32:554-60.

32. Ito $\mathrm{H}$, Taylor L, Bowman M, et al. Presentation and therapy of spontaneous coronary artery dissection and comparisons of postpartum versus nonpostpartum cases. Am J Cardiol 2011;107: 1590-6.

Citation: Ibrahim Osman, OlisaemekaAchike, Dan Le, Assad Movahed. "Unusual Distal Lad Spiral Dissection in Young Male". American Research Journal of Clinical Case Reports; 1(1): 2-13.

Copyright (c) Ibrahim Osman, OlisaemekaAchike, Dan Le, Assad Movahed. This is an open access article distributed under the Creative Commons Attribution License, which permits unrestricted use, distribution, and reproduction in any medium, provided the original work is properly cited. 